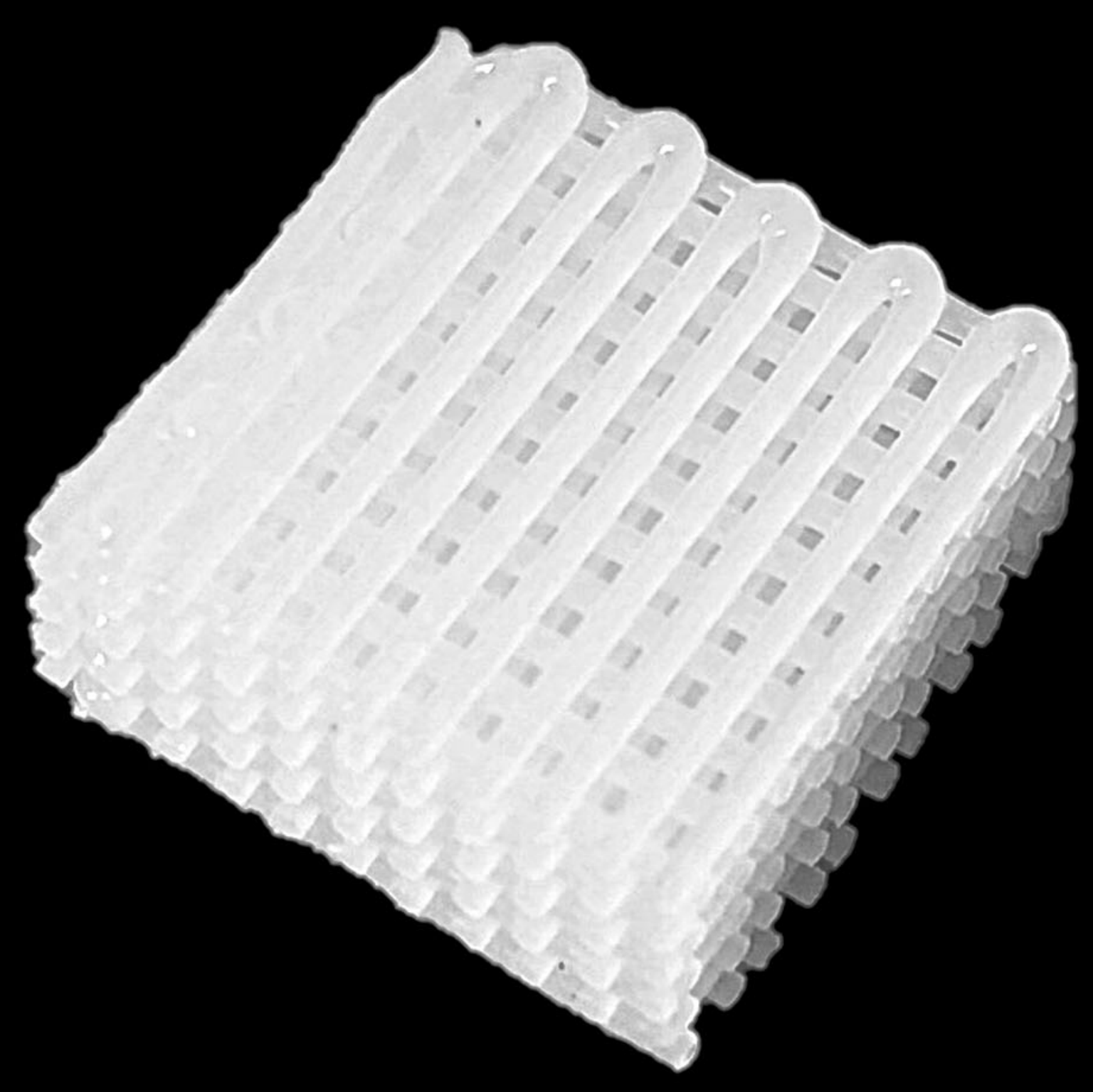




\title{
Hybrid Additive Manufacturing System for Zonal Plasma-Treated Scaffolds
}

\author{
AU1 Fengyuan Liu. * Weiguang Wang, Srichand Hinduja, and Paulo Jorge Bártolo*
}

\begin{abstract}
Producing synthetic scaffolds with adequate physical, chemical, and biological properties remains a challenge for tissue engineering. Internal architecture, surface chemistry, and material properties have strong impact on the cell biological behavior. This requires sophisticated systems not only able to process multiple materials with different characteristics, creating fully interconnected 3D porous structures with high reproducibility and accuracy, but also able to modify their properties during the fabrication process. This study introduces a novel additive manufacturing system comprising a multiprinting unit (screw-assisted and pressure-assisted printing heads) together with a plasma unit that enables the surface modification of printed scaffolds. Poly $\left(\varepsilon^{-}\right.$ caprolactone) scaffolds with a lay-down pattern of $0 / 90^{\circ}$ were fabricated using the screw-assisted printing head and a plasma jet unit used to uniformly modify each layer, a specific region of each layer during the printing process or the external surface of the printed scaffolds. Scaffolds were produced using different plasma exposure times and different distance between the plasma head and the printed layer, and fixed printing conditions. Produced scaffolds were morphologically, mechanically, chemically, and biologically characterized. Results show that the distance between the plasma head and the printed material has no significant effect on the mechanical properties, whereas the increase of the plasma deposition velocity increases the mechanical properties. As expected, plasma treatment increases hydrophilicity and consequently the biological performance of the scaffolds. Results also show the potential of the proposed fabrication system to create functional gradient or scaffolds with tailored properties.
\end{abstract}

Keywords: additive manufacturing, plasma-assisted bioextrusion system, plasma surface modification, scaffold, tissue engineering

\section{Introduction}

Tissue ENGINEERING is a multidisciplinary field for the development of biological substitutes that restore, maintain, or improve tissue function by combining cells with three dimensional (3D) porous scaffolds. ${ }^{1,2}$ In this field, the fabrication of $3 \mathrm{D}$ highly porous, biocompatible, and biodegradable scaffolds, with proper porosity, pore size, shape and interconnectivity, degradation rate, chemical composition, and mechanical properties, is critical.

Recently, additive manufacturing technologies have emerged as a promising approach to produce scaffolds. ${ }^{2-6}$ The main advantages they provide are the capacity to rapidly produce very complex 3D constructs in a layer-by-layer manner and the ability to actively design the porosity and interconnectivity of scaffolds enabling repeatable macroarchitectures. However, low resolution limits the fabrication of scaffolds to those with large pores compared with the size of cells. This leads to low cell-seeding efficiency and a nonuniform distribution of cells, and consequently to low rates of tissue formation, less uniform tissue, and different cell differentiation behavior. ${ }^{7,8}$ Moreover, most additive fabrication techniques are usually limited to the fabrication of single material scaffolds, which may not be fully chemically compatible with cells. ${ }^{9}$ Furthermore, cell adhesion in the core regions of these scaffolds is often obstructed by the tortuosity of the scaffold, leading to limited cell colonization. ${ }^{10} \mathrm{In}$ addition, as synthetic biopolymers, the most commonly used

\footnotetext{
AU2 School of Mechanical, Aerospace and Civil Engineering, The University of Manchester, Manchester, United Kingdom.
}

Correct *Cofirst authors, contributed equally to this work.

Opposite page: Hybrid additive manufacturing system for zonal plasma treated scaffolds. Photo Credit: Dr. Fengyuan Liu.

The legend for the splash image should be corrected into 'Zonal plasma treated PCL scaffold fabricated with a hybrid additive manufacturing system'. 
PE-CVD: plasma-enhanced chemical vapor deposition

materials, are hydrophobic, limited cell adhesion and proliferation are observed. ${ }^{7}$

As cells are sensitive to the surface characteristics and topography of surfaces, surface-modification techniques such as

AU3 cold plasma deposition (PE-CVD), etching, and grafting processes, can be effective in tailoring the morphology and properties of surfaces to improve and orient their interaction with cells. ${ }^{4,11}$ In particular, PE-CVD of stable functionalized coatings (i.e., characterized by surface functional groups such as $-\mathrm{NH}_{2}$, $-\mathrm{COOH}$, and others) and plasma grafting of polar groups allow the hydrophilic character of surfaces to be enhanced. ${ }^{12-14}$ This provides anchor groups for biomolecule immobilization, which ultimately improve cell adhesion, colonization, and growth.

Despite its efficiency on the treatment of 2D substrates, the application of plasmas for the homogeneous modification of 3D scaffolds remains unviable due to limited penetration depth. Cold plasmas at low pressure (10-1000 mTorr), the most versatile plasma processes, are not ignited in $10-400 \mu \mathrm{m}$ pores. ${ }^{15}$ In these conditions, plasma active species can reach the walls of the pores only by diffusion. ${ }^{16}$ At atmospheric pressure, it may be possible to make some progress with dielectric barrier discharges, or plasma jets, but the problem of limited penetration in a 3D scaffold remains.

This article presents a novel additive manufacturing system, named as plasma-assisted bioextrusion system (PABS), aiming to radically move behind the current state of the art to circumvent the previously mentioned problems by developing a plasma-integrated hybrid additive manufacturing system. ${ }^{17}$ The system operates both the fabrication and the plasma treatment in a layer-by-layer manner, allowing highly efficient use of plasma 2D surface treatments, overcoming challenges related to full-cell penetration and colonization.
Moreover, this system opens up the possibility of tailoring the plasma treatment for particular areas of the scaffolds. To assess the system, poly ( $\varepsilon$-caprolactone) (PCL) scaffolds were produced and plasma treated considering three strategies: surface treated, center treated, and full-layer treated. The effect of plasma exposure time (i.e., plasma deposition velocity) and intensity (i.e., distance between plasma head and scaffold) was investigated. Hydrophilicity changes under different plasma conditions were determined with water contact angle measurement and compression tests were carried out to evaluate the effect of plasma treatment on the mechanical properties of the scaffolds. In vitro biological assessments were conducted to assess the adhesion and proliferation behavior of human adipose-derived stem cells (hADSCs) on fabricated scaffold samples (untreated, surfacetreated, center-treated, full-layer-treated PCL scaffolds).

\section{Materials and Methods}

Poly( $\varepsilon$-caprolactone)

PCL $\left(\mathrm{CAPA}^{\mathrm{TM}} 6500, \mathrm{Mw}=50,000 \mathrm{~g} / \mathrm{mol}\right)$, purchased from Perstorp (Cheshire, United Kingdom) in the form of $3 \mathrm{~mm}$ pellets, was used to produce the scaffolds. PCL is an easy-toprocess semicrystalline polymer with a density of $1.1 \mathrm{~g} / \mathrm{cm}^{3}$, a melting temperature between $58^{\circ} \mathrm{C}$ and $60^{\circ} \mathrm{C}$, and a glass transition temperature of $-60^{\circ} \mathrm{C}$.

\section{Experiment setup}

The PABS setup, shown in Figure 1a, allows the fabrication of multimaterial scaffolds with either uniform pore size or gradient pore size, specially controlled mechanical

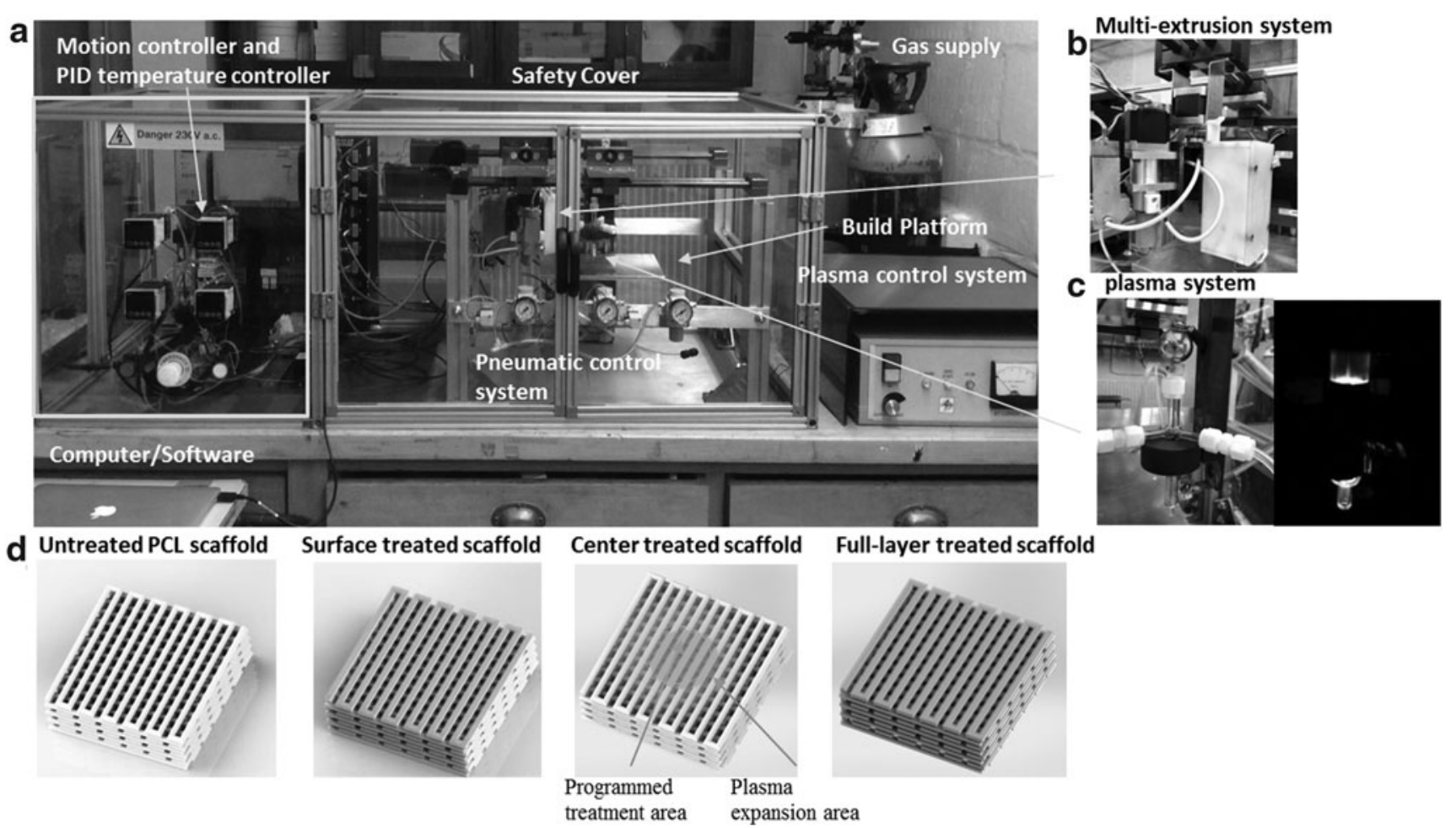

FIG. 1. (a) Setup of plasma-assisted bioextrusion system; (b) multiextrusion system; (c) plasma modification system; (d) schematic images of untreated PCL scaffolds, surface-treated scaffolds, center-treated scaffolds, and full-layer-treated scaffolds. PCL, poly(e-caprolactone). 
properties and chemical homogeneous and heterogeneous surface to modulate cell adhesion, proliferation, and differentiation. The system comprises two main units, a multiextrusion printing system (Fig. 1b) and a plasma jet system (Fig. 1c). The multiextrusion system comprises two pressureassisted printing heads and one screw-assisted printing head, allowing to operate with a range of thermoplastic biomaterials, hydrogels, and thermoplastic/ceramic materials. All printing heads are equipped with a heated material chamber operating with a set of nozzle diameters ranging from 0.1 to $1 \mathrm{~mm}$. The plasma jet head consists of a quartz capillary with a pin-type electrode mounted in coaxial geometry at the center of the capillary, and a ground electrode outside. Different types of gases can be applied. The control software was developed in MATLAB as previously reported and generated $\mathrm{G}$ code files contains all the instructions for the fabrication process. ${ }^{18}$

\section{Scaffold design and fabrication}

Rectangular scaffolds of $12 \times 12 \times 3 \mathrm{~mm}^{3}$ (length $\times$ width $\times$ height) were designed with a lay-down pattern of $0 / 90^{\circ}$ and filament distance of $1 \mathrm{~mm}$. The printing conditions were deposition rate of $3 \mathrm{~mm} / \mathrm{s}$, slice thickness of $0.5 \mathrm{~mm}$, heating temperature of $90^{\circ} \mathrm{C}$, extrusion rate of $15 \mathrm{rpm}$, and nozzle tip size of $0.5 \mathrm{~mm}$.

\section{Plasma modification strategies and processing parameters}

The plasma treatment was performed using nitrogen $\left(\mathrm{N}_{2}\right)$ at a pressure of $10 \mathrm{psi}$ with a flow rate of $5 \mathrm{~L} / \mathrm{mm}$ directly exposed to selected areas of the scaffolds in atmospheric environment. Tests were performed at different deposition velocities (3, 6, and $12 \mathrm{~mm} / \mathrm{s})$, and at different distances between the plasma unit and the surface of the deposited material $(5,10$, and $15 \mathrm{~mm})$. High voltage $(10 \mathrm{kV})$ and radiofrequency (repetition rate $=50 \mathrm{KHz}$ ) were ignited between the tungsten electrode and copper film. Three different strategies were considered (Fig. 1d):

(1) Surface-treated scaffolds: PCL scaffolds were printed using the screw-assisted printing head and then uniformly treated with the plasma system.

(2) Center-treated scaffolds: PCL scaffolds were printed and, during the printing process, the center of each layer was treated with the plasma system. The centertreated area is determined by the programmed tool path of the plasma jet, which was set as a circular area with $5 \mathrm{~mm}$ diameter. For short treatment distances (e.g., $5 \mathrm{~mm}$ ), the treatment area is approximately the same as the programmed area. Currently, the plasma exposure area is controlled by adjusting the distance between the plasma system and the material surface, with the other parameters (e.g., power and deposition velocity) enabling to control the degree of surface modification. An improvement on the plasma jet is being considered by creating a well-defined gas curtain around the reaction jet to shield the effluent and get it focused. 19

(3) Full-layer-treated scaffolds: The screw-assisted printing head was used to print a layer of PCL and then the plasma system was used to treat the layer.

\section{Morphological characterization}

Scanning electron microscope (SEM) was used to assess the morphology and surface characteristic of both plasmatreated and untreated scaffolds. The scaffolds were gold/ palladium coated using a Q150T turbo-pumped sputter coater (Quorum Technologies, United Kingdom) and imaged at $10 \mathrm{kV}$ (Hitachi S3000N, Japan). These images were then analyzed using ImageJ software.

\section{Chemical characterization}

The X-ray photoelectron spectroscope (XPS) analysis was performed using a Kratos Axis Ultra (Kratos Analytical Ltd, Manchester, United Kingdom) with CasaXPS software for data acquisition and analysis. The monochromatic $\mathrm{Al}(\mathrm{K} \alpha)$ $\mathrm{X}$-ray source ( $\mathrm{hv}=15 \mathrm{kV} ; 300 \mathrm{~W}$ set power) was used. The spectrometer was operated in constant analyzer energy mode with a pass energy of $100 \mathrm{eV}$ for survey scan, whereas for the carbon $(\mathrm{C}) 1 \mathrm{~s}$, oxygen $(\mathrm{O}) 1 \mathrm{~s}$, and nitrogen $(\mathrm{N}) 1 \mathrm{~s}$, individual high-resolution spectra were taken at a pass energy of 50 and a $0.1 \mathrm{eV}$ energy step. The binding energy $(\mathrm{eV})$ scales were referenced to the hydrocarbon component $(\mathrm{C}-\mathrm{C})$ in the $\mathrm{C} 1 \mathrm{~s}$ spectra at $285 \mathrm{eV}$.

\section{Surface wettability characterization}

Water contact angle measurements on the surfaces of untreated and plasma-treated PCL scaffolds were carried out with a KSV CAM 200 system (KSV Instruments, Finland). The system is equipped with a CCD video camera and a micrometric liquid dispenser to drop $2 \mu \mathrm{L}$ of distilled water on the surface of the scaffold. The contact angle measurements were automatically calculated with the instrument software. The water droplet was deposited at the center of the scaffold surface for each sample.

\section{Mechanical characterization}

Compression tests were performed to analyze the effect of plasma processing parameters on the mechanical properties of fully plasma-treated PCL scaffolds. Before the compression test, the edges of all scaffolds were vertically cut with a razor blade creating a cross-section of $\sim 10 \times 10 \mathrm{~mm}$. All tests were carried out using an unconfined Instron 3344 single column universal testing systems (Instron Corporation, Buckinghamshire, United Kingdom) equipped with a $2000 \mathrm{~N}$ load cell. Specimens were compressed between two fixed stainless steel plates at a rate of $0.5 \mathrm{~mm} / \mathrm{min}$ up to a strain value of $0.45 \mathrm{~mm} / \mathrm{mm}$. For each type of specimen, the tests were repeated four times in quadruplicate. Data were collected and analyzed using the OriginPro 8.5.1 software (OriginLab Corporation). The stress $(\sigma)$ was evaluated as the ratio between the load $(F)$ and the total area $(A)$ of the apparent cross section of the scaffold according to the following equation:

$$
\sigma=\frac{F}{A}
$$

whereas strain, $\varepsilon$, was defined as the ratio between the scaffold height variation $\Delta h$ (the difference between initial height 
and the height after compression) and the scaffold initial height $h_{0}$ :

$$
\varepsilon=\frac{\Delta h}{h_{0}}
$$

The compressive modulus was defined as the slope of the tangent line to the stress-stain curve at $12.5 \%$ strain.

\section{Biological assessment}

Cell proliferation tests were performed using hADSCs (STEMPRO, Invitrogen, Waltham, MA). Before cell seeding, scaffolds were sterilized by soaking in $70 \%$ ethanol for $2 \mathrm{~h}$. After sterilization, samples were rinsed twice with phosphatebuffered saline (PBS) (Gibco, ThermoFisher Scientific, Waltham, MA), transferred to 24 -well plates and air-dried for $24 \mathrm{~h}$ at room temperature. A total of 50,000 cells were seeded on each sample, including untreated scaffolds, plasma surfacetreated, center-treated, and full-layer-treated scaffolds.

Cell viability/proliferation behavior and the percentage of cells attached on the scaffolds (cell-seeding efficiency) were assessed through Alamar Blue assay (also termed the Resazurin assay, reagents from Sigma-Aldrich, Dorset, United Kingdom). Cell viability/proliferation rate was measured at $1,3,7$, and 14 days after cell seeding. For each measurement, cell-seeded scaffolds were transferred to a new 24-well plate and $0.7 \mathrm{~mL}$ of Alamar Blue solution was added to each well; the plate was incubated for $4 \mathrm{~h}$ under standard condition $\left(37^{\circ} \mathrm{C}, 5 \% \mathrm{CO}_{2}\right.$, and $95 \%$ humidity). After incubation, $150 \mu \mathrm{L}$ of each sample solution was transferred to a 96-well plate and the fluorescence intensity was measured at $540 \mathrm{~nm}$ excitation wavelength and $590 \mathrm{~nm}$ emission wavelength with a spectrophotometer (Sunrise, Tecan, Männedorf, Zurich, Switzerland).

Cell attachment and distribution are assessed using laser confocal microscopy, with cell nuclei stained. At day 1 of cell culture and after cell culture for 14 days, scaffolds were removed from the 24-well cell culture plate, rinsed twice in PBS (Gibco, ThermoFisher Scientific, Waltham, MA), and fixed with $10 \%$ neutral buffered formalin (Sigma-Aldrich) for $30 \mathrm{~min}$ at room temperature. After fixation, samples were rinsed twice with PBS for the removal of formalin, then permeabilized with $0.1 \%$ Triton-X100 (Sigma-Aldrich) in PBS at room temperature for $10 \mathrm{~min}$, and rinsed twice for the removal of Triton-X100. Cell nuclei were stained blue by soaking scaffolds in a PBS solution containing 4',6diamidine-2'-phenylindole dihydrochloride (Sigma-Aldrich) at the manufacturer-recommended concentration. Samples were left in the staining solution for $10 \mathrm{~min}$ before removal and rinsed twice thoroughly with PBS. Confocal images were obtained using a 3D rendering mode on a Leica TCS SP5 (Leica, Milton Keynes, United Kingdom) confocal microscope, and cell colonization was quantified using a standard z-stack method. All images were taken at the center area of the scaffolds and the experiments were performed three times in triplicate.

\section{Data analysis}

All data are represented as mean \pm standard deviation. Biological results were subjected to one-way analysis of variance (one-way ANOVA) and post hoc Tukey's test using GraphPad Prism software. Significance levels were set at $p<0.05$.

\section{Results}

\section{Morphological characterization}

SEM images of full-layer plasma-treated PCL scaffolds are shown in Figure 2a. As seen from the top view and side view of the SEM images, the structure presented an interconnected structure with pores of $\sim 500 \mu \mathrm{m}$ uniformly distributed. Figure $2 \mathrm{~b}$ shows the top view of a filament surface from untreated and plasma-treated scaffolds. The surface of untreated filaments (Fig. 2b (left)) exhibited a nonsmooth morphology with small micropores ( $\sim 5-20 \mu$ m diameters), whereas the surface of $\mathrm{N}_{2}$ plasma-treated filaments shows a denser and smaller nano-size roughness with lines in the direction of the plasma jet movement (Fig. 2b (匹eft)).

\section{Chemical characterization}

$$
\text { change to 'right' }
$$

The chemical composition of untreated and plasma-treated scaffolds, evaluated by XPS, is presented in Table 1 and Figure $2 \mathrm{c}$ and $\mathrm{d}$. Results show that after plasma modification, the shape of the $\mathrm{C} 1 \mathrm{~s}$ peak dramatically changed with the increase in both $\mathrm{C} 2$ and $\mathrm{C} 3$, and the appearance of amide groups $(\mathrm{N}-\mathrm{C}(=\mathrm{O}), \mathrm{C} 5)$, confirming that the PCL chemical composition was modified with an $\mathrm{N}$-rich coating.

\section{Surface wettability characterization}

Figure $3 \mathrm{a}$ shows that the untreated PCL scaffolds present higher water contact angle $\left(85.48^{\circ} \pm 1.5^{\circ}\right)$, typical of hydrophobic surfaces. The figure also revealed a slightly lower water contact angle of full-layer-treated PCL scaffolds $\left(59.07^{\circ} \pm 4.3^{\circ}\right)$ compared with surface-treated scaffolds $\left(62.56^{\circ} \pm 2.7^{\circ}\right)$. Moreover, the center-treated scaffold presented lowest water contact angle $\left(52.04^{\circ} \pm 2.4^{\circ}\right)$ in comparison with the full-layer and surface-treated scaffolds.

The effect of plasma processing parameters, such as the distance between the plasma head and deposited material and plasma deposition velocity on the surface wettability, was also investigated, considering full-layer-treated scaffolds. Figure $3 \mathrm{~b}$ shows the water contact angle measurements after water dropped on the surface for 0,3 , and $5 \mathrm{~s}$ for samples treated with the same deposition velocity of $3 \mathrm{~mm} / \mathrm{s}$, and different distances between the plasma jet and the deposited material. The results show that by increasing the distance the water contact angle decreases, which is associated with a decrease on the plasma effect. Figure $3 \mathrm{c}$ shows the effect of plasma deposition velocity, considering a $10 \mathrm{~mm}$ distance between the plasma head and the printed material. The results show that the plasma modification effect decreases with increasing plasma deposition velocity.

\section{Mechanical characterization}

Mechanical compression tests were performed to assess the effect of the plasma treatment processing conditions on the mechanical performance of the scaffolds. Obtained results are presented in Figure 4 and Tables 2 and 3. As observed, for a fixed distance between the plasma head and the deposited material, the plasma deposition velocity has a 
a

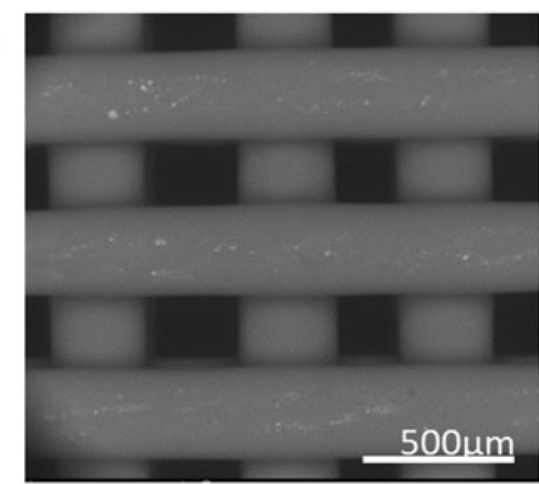

b
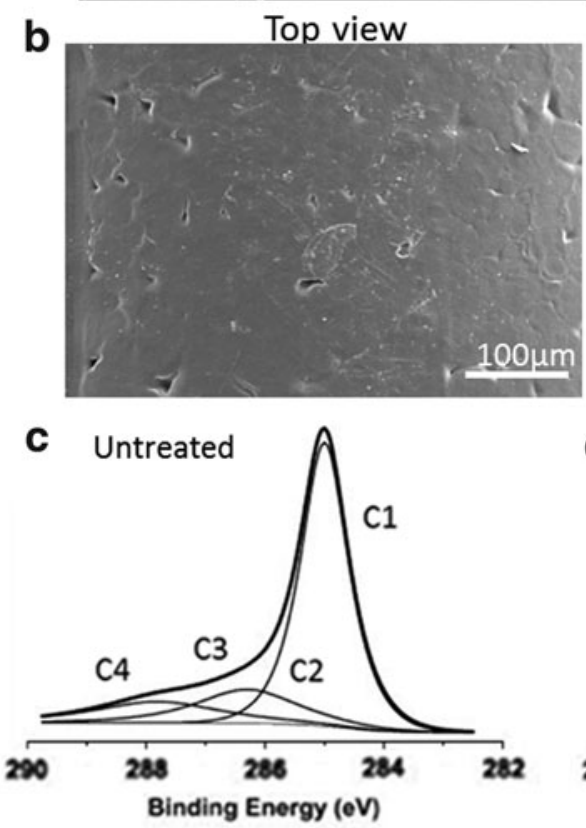

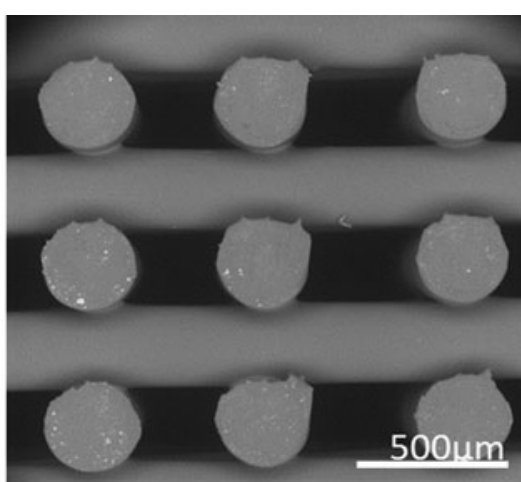

Side view
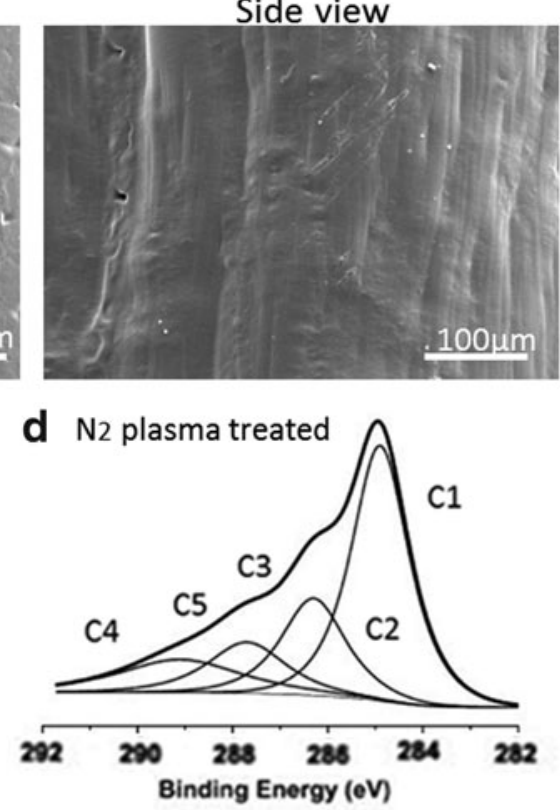

AU9 FIG. 2. SEM images of (a) top view and cross section view of 3D printed PCL scaffolds after $\mathrm{N}_{2}$ plasma treatment, scale bar $500 \mu \mathrm{m}$; (b) filament surfaces of untreated and plasma-treated scaffolds, scale bar $100 \mu \mathrm{m}$. SEM, scanning electron microscope.

In figure 2, after current legend, please include ' The C1s spectra of (c) untreated and (d) N2 plasma-treated PCL scaffolds'.

significant effect on the mechanical properties, with the compression modulus varying between $21.41 \mathrm{MPa}$ at $3 \mathrm{~mm} / \mathrm{s}$ and $34.30 \mathrm{MPa}$ at $12 \mathrm{~mm} / \mathrm{s}$. For a fixed plasma deposition velocity, the effect of changing the distance between the plasma head and the deposited material on the compressive modulus is less significant.

Table 1. The Concentration of Carbon, Oxygen, and Nitrogen ON Untreated SCAFFold and $\mathrm{N}_{2}$ Plasma-Treated Scaffolds

(10 mm Treatment Distance and $3 \mathrm{~mm} / \mathrm{s}$ Plasma Deposition Velocity) as Determined by X-Ray Photoelectron Spectroscope Analysis

\begin{tabular}{lcc}
\hline Element & Untreated & $N_{2}$ plasma treated \\
\hline C\% & $76.7 \pm 1$ & $72.4 \pm 1$ \\
O\% & $23.3 \pm 1$ & $22.6 \pm 1$ \\
N\% & 0 & $5 \pm 1$ \\
C-C\% & $52.4 \pm 1$ & $53.4 \pm 1$ \\
-C-N-\% & 0 & $4.4 \pm 1$ \\
-C-O-\% & $19.2 \pm 1$ & $18.3 \pm 1$ \\
\hline
\end{tabular}

\section{Biological assessment}

Cell adhesion and proliferation of four types of 3D scaffolds $\left(\mathrm{N}_{2}\right.$ plasma surface treated, center treated, full-layer treated, and untreated) are assessed using the Alamar Blue assay. Fluorescence intensity of cell-seeded scaffolds was measured at four different culture time points $(1,3,7$, and 14 days), as presented in Figure 5a. The results show that for both plasma-treated and untreated scaffolds, the fluorescence intensity increases from one time point to another, suggesting that the scaffolds fabricated with the developed additive manufacturing system are suitable substrates for cell proliferation. At both day 1 and day 3, no significant differences were observed between the different types of scaffolds. However, at day 7, plasma surface-treated and full-layertreated scaffolds present statistically higher fluorescence intensity than untreated scaffolds, suggesting higher cell attachment/proliferation rate due to the plasma surface modification. At day 14, plasma surface-treated scaffolds show statistically higher cell proliferation than both untreated scaffolds and center-treated scaffolds. No statistical differences were observed between plasma surface-treated and full-layer-treated scaffolds. 
Figure 3 legend corrected into: ' (a) water contact angles of untreated, surface-treated, center-treated, and full-layer-treated PCL scaffolds after the water drop on the surface at $0 \mathrm{~s}$; effect of (b) the distance from plasma jet on the deposited material (at deposition velocity of $3 \mathrm{~mm} / \mathrm{s}$ ), and (c) the plasma deposition velocity (with $10 \mathrm{~mm}$ distance) on the surface wettability detected by water contact angle results at time points 0,3 , and $5 \mathrm{~s}$ '.
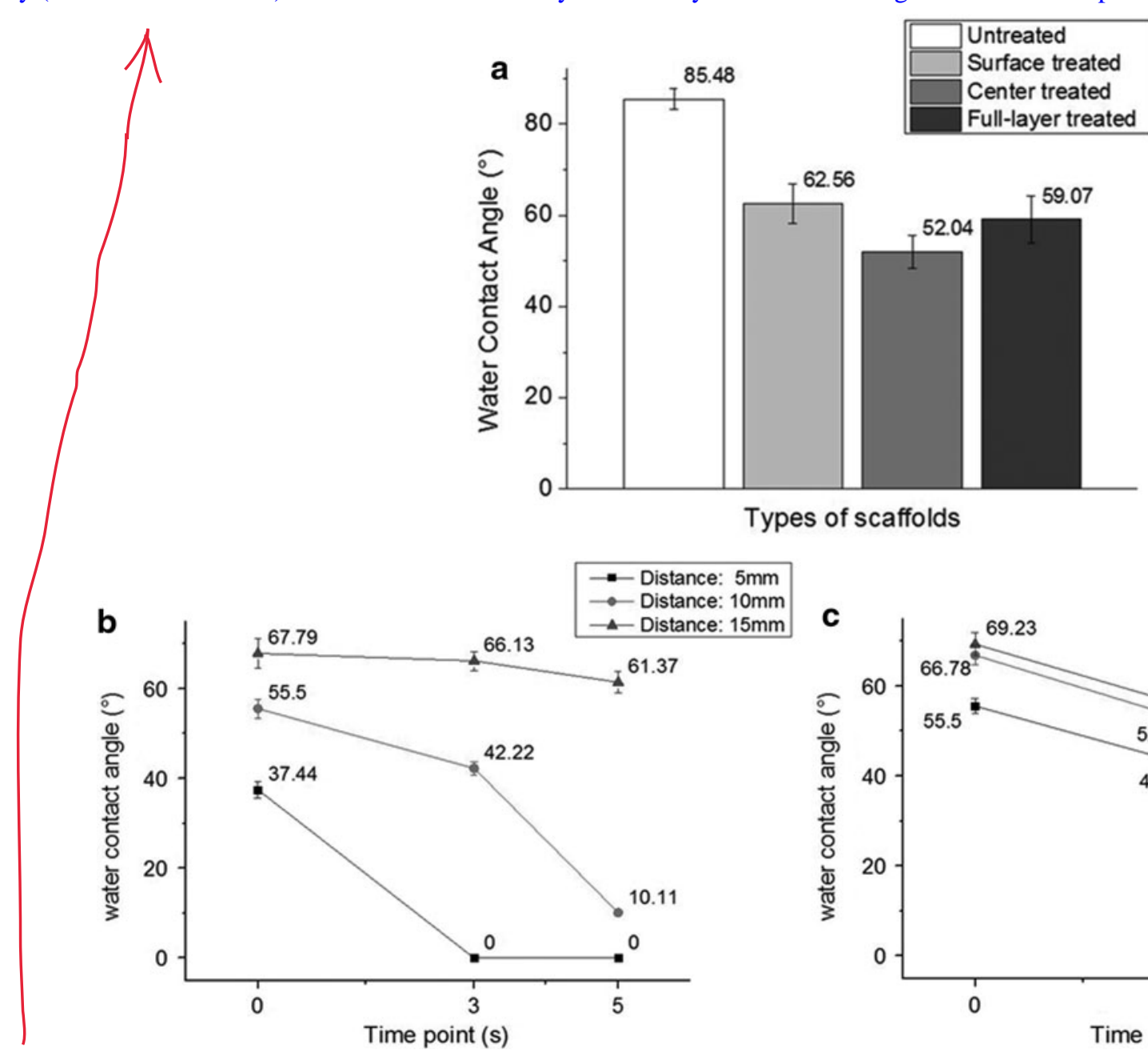

AU10 FIG. 3. The C1s spectra of (a) untreated and (b) $\mathrm{N}_{2}$ plasma-treated PCL scaffolds; (c) water contact angles of untreated, surface-treated, center-treated, and full-layer-treated PCL scaffolds after the water drop on the surface at $0 \mathrm{~s}$; effect of (d) the distance from plasma jet on the deposited material (at deposition velocity of $3 \mathrm{~mm} / \mathrm{s}$ ), and (e) the plasma deposition velocity (with $10 \mathrm{~mm}$ distance) on the surface wettability detected by water contact angle results at time points 0,3 , and $5 \mathrm{~s}$.

a

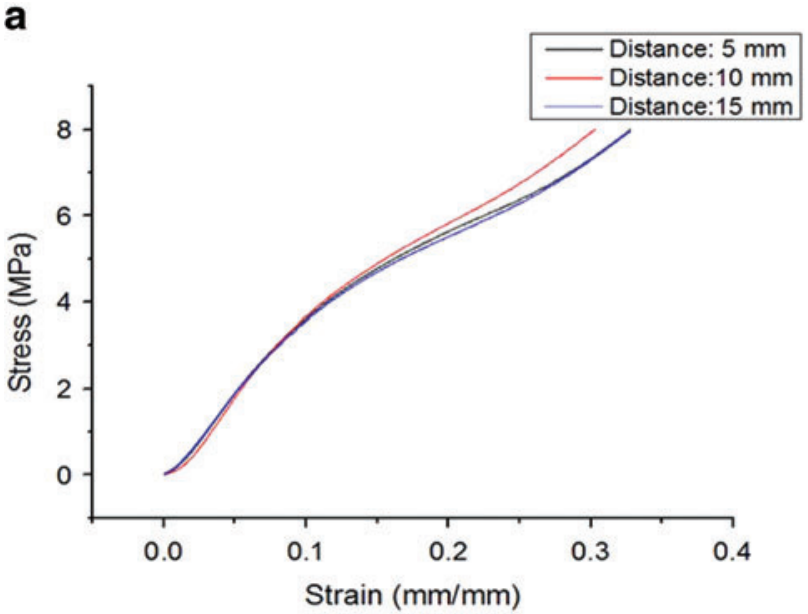

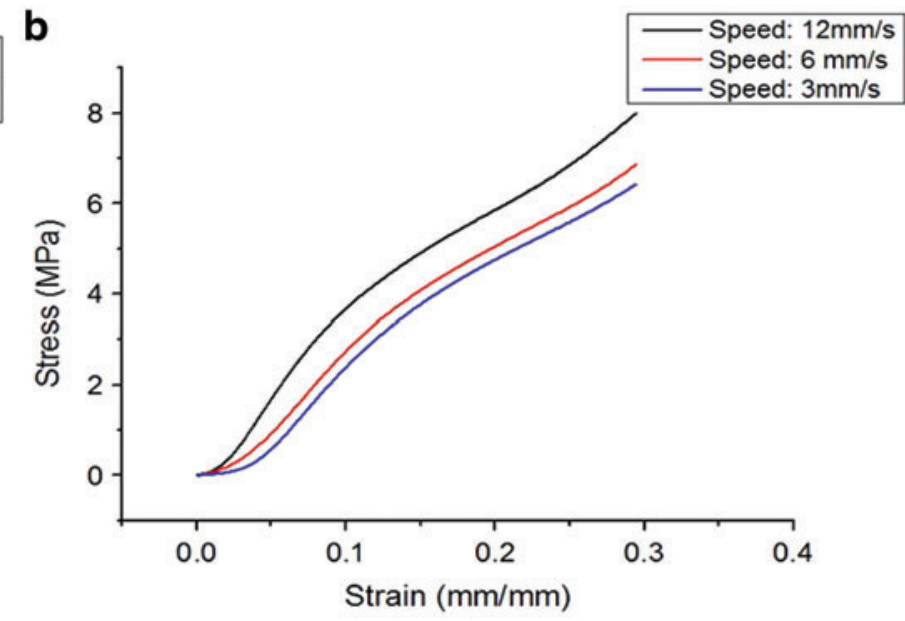

b

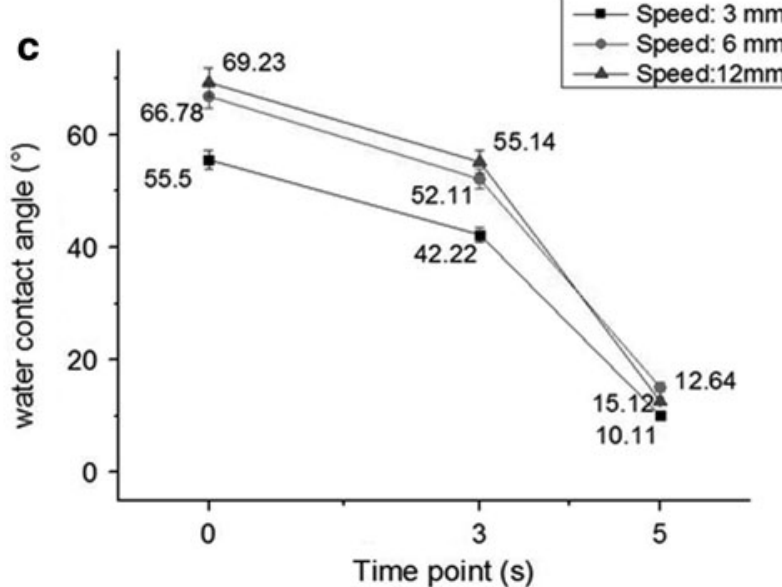

FIG. 4. Effect of full-layer plasma modification on the compressive mechanical properties with (a) different distance between plasma jet and deposited material (at velocity of $3 \mathrm{~mm} / \mathrm{s}$ ) and (b) plasma deposition velocity (with $10 \mathrm{~mm}$ distance). Typical stress-strain curves for PCL scaffolds characterized by a $0 / 90^{\circ}$ lay-down pattern and filament distance of $500 \mu \mathrm{m}$, compressed at a rate of $0.5 \mathrm{~mm} / \mathrm{min}$ up to a strain value of $0.4 \mathrm{~mm} / \mathrm{min}$. Color images available online at www.liebert pub.com/3dp 
Table 2. Compressive Modulus for Plasma-Treated

Scaffolds With Different Treatment Distances and a Constant Plasma Deposition Velocity of $3 \mathrm{~mm} / \mathrm{s}$

\begin{tabular}{lccc}
\hline & \multicolumn{3}{c}{$\begin{array}{c}\text { Plasma treatment distance } \\
\text { between plasma head } \\
\text { and deposited material }(\mathrm{mm})\end{array}$} \\
\cline { 2 - 4 } & 5 & 10 & 15 \\
\hline $\begin{array}{c}\text { Compressive } \\
\text { modulus }(\mathrm{MPa})\end{array}$ & $22.55 \pm 1.2$ & $21.41 \pm 0.8$ & $23.23 \pm 1.4$ \\
\hline
\end{tabular}

Confocal microscopy images (Fig. 5b) present the cell attachment and distribution after cell seeding (day 1) and proliferation (day 14). It can be observed that plasma-treated scaffolds presented higher number of cells than untreated scaffolds. In addition, it is also possible to observe that plasma surface-treated scaffolds presented best cell attachment and dispersion.

\section{Discussion}

The SEM results indicate that the plasma surface modification has an impact on the surface roughness, with the results focusing on the extruded filament confirming this impact. The micropores observed on the surface of untreated filament are due to the structure evolution during the screw-assisted melt extrusion process. ${ }^{20}$ After plasma treatment, the denser surface appearance indicates an increase in the surface roughness due to the etching process, during which the melting and etching consecutively occurred on the PCL surface. ${ }^{21,22}$ This etching results in the stripping off the topmost layer of the polymer due to the weight loss during the plasma exposure. ${ }^{16}$ Moreover, the textures on the treated filament surface are attributed to the gas flow generated by the plasma jet during the modification process. In this case, as the printed material is not totally cooled down, when the plasma modification occurs, the gas flow effect is stronger, significantly influencing the surface topography.

AU4 The WCA measurement results indicate that $\mathrm{N}_{2}$ plasma WCA: Water modification dramatically increased the wettability of PCL scaffolds. The effect of the chemical groups deposited in the bottom layers of full-layer-treated scaffolds enhanced the better hydrophilicity behavior than surface-treated scaffolds. Since the center treatment guided the direction of water absorption in the vertical direction, limiting the spreading in the horizontal direction, the center-treated scaffolds present time-proved wettability. The effect of plasma modification can be tailored by changing the plasma processing parameters, including the distance between the plasma head and

Table 3. Compressive Modulus for Plasma-Treated Scaffolds With Different Deposition Velocities and a Constant Plasma Treatment Distance of $10 \mathrm{Mm}$

\begin{tabular}{lccc}
\hline & \multicolumn{3}{c}{$\begin{array}{c}\text { Plasma deposition } \\
\text { velocity }(\mathrm{mm} / \mathrm{s})\end{array}$} \\
\cline { 2 - 4 } & 3 & 6 & 12 \\
\hline $\begin{array}{c}\text { Compressive } \\
\text { modulus (MPa) }\end{array}$ & $21.41 \pm 0.8$ & $26.14 \pm 1.2$ & $34.30 \pm 1.5$ \\
\hline
\end{tabular}

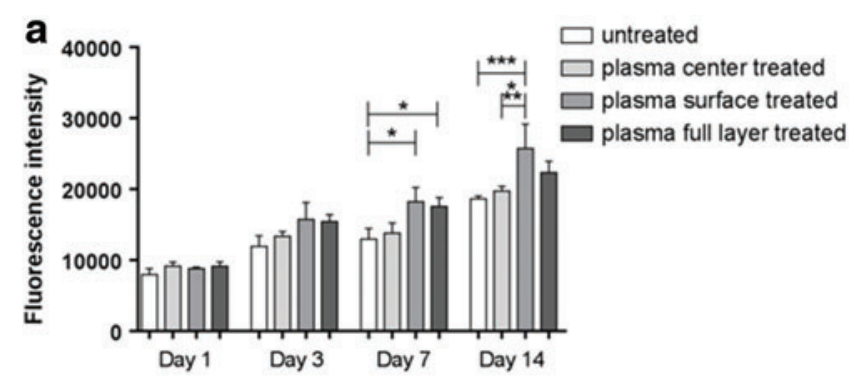

b

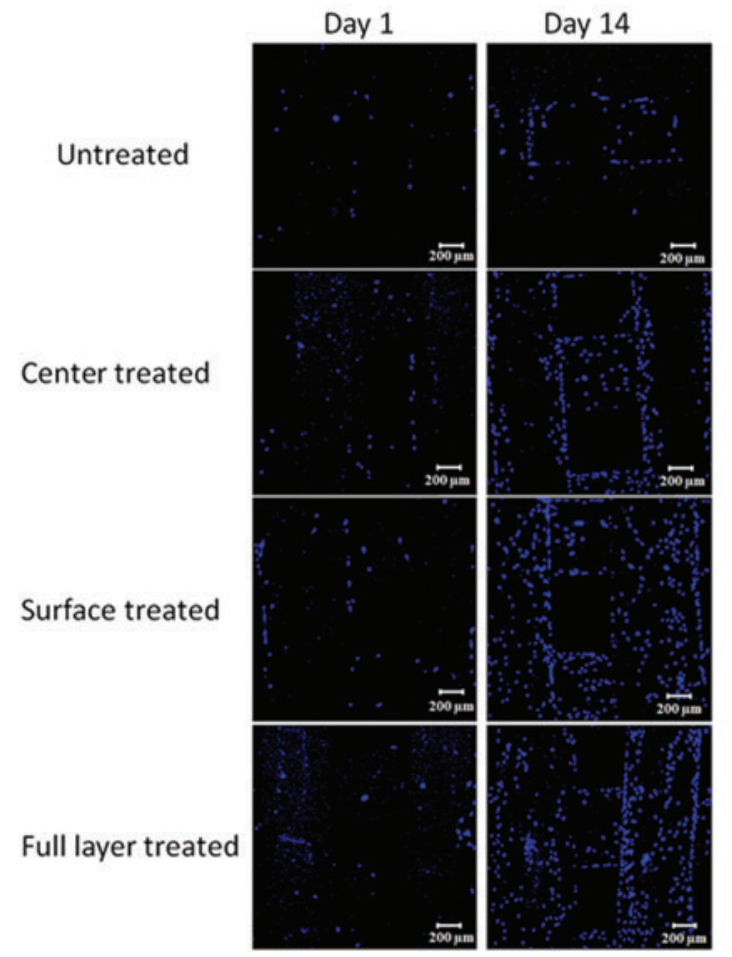

FIG. 5. (a) Cell viability/proliferation test result (fluorescence intensity) of cell-seeded PCL scaffolds (untreated and $\mathrm{N}_{2}$ plasma center, surface, and full-layer treated) at days 1 , 3,7 , and 14 of cell culturing; (b) confocal microscope images of all types of cell-seeded scaffolds, 1 day and 14 days after cell culture. Scale bar $250 \mu \mathrm{m}$. Color images available online at www.liebertpub.com/3dp

deposited material, and plasma deposition velocity. The plasma modification effect is related to the spatial distribution of active species and temperature and also the pressure associated with the plasma treatment. ${ }^{23,24}$ For smaller distances, the higher temperature, higher pressure, and more concentrated active species induce lower WCA on the treated surface. Higher plasma deposition velocity results in the shorter plasma exposure time, which leads to less active species deposited on the surface.

The effect of plasma modification process on the mechanical properties is further investigated in this study by changing the plasma processing parameters. The mechanical analyses revealed that with a constant plasma modification velocity, the distance does not affect the bulk properties, which is consistent with previous studies, ${ }^{16,25}$ whereas the plasma modification velocity has an effect on the compressive behavior. Results show that slower plasma deposition velocities resulted in reduced compressive modulus. Since the deposited material 
is not fully solidified when conducting the plasma modification, lower plasma velocities may influence the microstructure development contributing to reduced mechanical properties. ${ }^{20,26}$

According to other studies, ${ }^{16,25}$ a significant increase in the biological behavior is observed after plasma modification. Owing to the zonal plasma modification capability of PABS, the biological behavior was further assessed through comparison of surface-treated, center-treated, and full-layertreated scaffolds. The center-treated scaffolds presented insignificantly improved results as the space for cell proliferation is limited in the core region of scaffolds. This also explained the higher fluorescence intensity observed for surface-treated and full-layer-treated scaffolds. Furthermore, the full-layer-treated scaffolds resulted in superhydrophilic properties, which may lead to decreased cell attachment and proliferation, ${ }^{27}$ and this explains the observed lower cell intensity than surface-treated scaffolds.

\section{Conclusions}

This article presented a novel additive manufacturing system comprising a multimaterial printing unit and a plasma jet unit. To assess the system, nonplasma-treated and plasmatreated PCL scaffolds were produced. The effect of plasma deposition velocity and the distance between the plasma head and the surface of the deposited material was investigated. Results from SEM analysis confirm the capability of the proposed system to produce full-layer or zonal plasma modified scaffold with fully interconnected channels and regular pore size. As observed, the filament morphology changed from a nonsmooth morphology with small micropores in the case of untreated scaffolds to a morphology with textures aligned with the direction of the plasma jet movement in the case of treated scaffolds. XPS results indicate the deposition of nitrogen-rich chemical groups on the top surface and throughout the entire $3 \mathrm{D}$ construct based on the treatment strategy. The water contact angle measurement results reveal that the scaffolds become more hydrophilic after the plasma treatment, and additionally, center-treated scaffolds appear to show the best hydrophilicity. Moreover, the effect of plasma treatment seems to be more significant with reduced deposition velocities and distances between the plasma head and the printed material. Mechanical compression tests show that for a fixed plasma deposition velocity, the effect of changing the distance between the plasma head and the deposited material is not significant. However, for a fixed distance, the compressive modulus decreases with the decrease in the plasma deposition velocity. In vitro biological assessment reveals better cell adhesion and proliferation behavior of hADSCs on the plasma-treated scaffolds, and the plasma surface-treated and plasma full-layer-treated scaffolds presented high biological performance. Bioimaging showing cell attachment and distribution also confirmed this observation.

Applications that may benefit from this technology include functional graded scaffolds, which have compositional variations tailored by plasma treatment with different gases, and various plasma deposition velocities and distances.

\section{Acknowledgment}

The authors wish to acknowledge the support from the School of Mechanical, Aerospace, and Civil Engineering at the University of Manchester.

\section{Author Disclosure Statement}

No competing financial interests exist.

\section{References}

1. Pereira RF, Barrias CC, Bártolo PJ, et al. Cell-instructive pectin hydrogels crosslinked via thiol-norbornene photoclick chemistry for skin tissue engineering. Acta Biomater 2017;66:282-293.

2. Vyas C, Pereira R, Huang B, et al. Engineering the vasculature with additive manufacturing. Curr Opin Biomed Eng 2017;2:1-13.

3. Kumar A, Mandal S, Barui S, et al. Low temperature additive manufacturing of three dimensional scaffolds for bone-tissue engineering applications: Processing related challenges and property assessment. Mater Sci Eng R 2016;103:1-39.

4. Wang M, Favi P, Cheng X, et al. Cold atmospheric plasma (CAP) surface nanomodified 3D printed polylactic acid (PLA) scaffolds for bone regeneration. Acta Biomater 2016;46: 256-265.

5. Wang W, Caetano GF, Chiang W-H, et al. Morphological, mechanical and biological assessment of PCL/pristine graphene scaffolds for bone regeneration. Int $\mathrm{J}$ Bioprint 2016;2:204-213.

6. Wang W, Caetano G, Ambler WS, et al. Enhancing the hydrophilicity and cell attachment of 3D printed PCL/ graphene scaffolds for bone tissue engineering. Materials 2016;9:992.

7. Oh SH, Lee JH. Hydrophilization of synthetic biodegradable polymer scaffolds for improved cell/tissue compatibility. Biomed Mater 2013;8:014101.

8. Sobral JM, Caridade SG, Sousa RA, et al. Three-dimensional plotted scaffolds with controlled pore size gradients: Effect of scaffold geometry on mechanical performance and cell seeding efficiency. Acta Biomater 2011;7:1009 1018

9. Boyd-Moss M, Fox K, Brandt M, et al. Bioprinting and Biofabrication with Peptide and Protein Biomaterials Peptides and Peptide-based Biomaterials and their Biomedical Applications. Springer, 2017; pp. 95-129.

10. Planell JA. Functional Scaffolds: Materials and Fabrication Congresso Nazionale Biomateriali-Lecce, 2012; p. 30.

11. Pertile RA, Andrade FK, Alves C, et al. Surface modification Milano, Italy. of bacterial cellulose by nitrogen-containing plasma for improved interaction with cells. Carbohydr Polym 2010;82: 692-698.

12. Surucu S, Masur K, Sasmazel HT, et al. Atmospheric plasma surface modifications of electrospun PCL/chitosan/ PCL hybrid scaffolds by nozzle type plasma jets for usage of cell cultivation. Appl Surf Sci 2016;385:400-409.

13. Roh HS, Jung SC, Kook MS, et al. In vitro study of 3D PLGA/n-HAp/ $\beta$-TCP composite scaffolds with etched oxygen plasma surface modification in bone tissue engineering. Appl Surf Sci 2016;388:321-330

14. Ghobeira R, De Geyter N, Morent R. Plasma surface functionalization of biodegradable electrospun scaffolds for tissue engineering applications. In: Biodegradable Polymers: Recent Developments and New Perspectives. IAPC Publishing, 2017.

15. Van Deynse A, Morent R, De Geyter N. Surface modification of polymers using atmospheric pressure cold plasma technology. In: Polymer Science: Research Advances, Practical Applications and Educational Aspects. Formatex Research Center, 2016; pp. 506-516. he publisher location is Gewerbestrasse 11, 6330 Cham, Switzerland.

4 AU5

AU6

The publisher location is

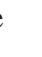
.

$\triangle \mathrm{AU} 7$

Editor name;Geraldine

Rohman

Location: Zagreb,

Croatia 
16. Intranuovo F, Gristina R, Fracassi L, et al. Plasma processing of scaffolds for tissue engineering and regenerative medicine. Plasma Chem Plasma Process 2016;36:269-280.

17. Liu F, Wang W, Bartolo P, et al. A plasma-assisted bioextrusion system for tissue engineering. CIRP Ann Manuf Technol 2018 (in press).

18. Liu F, Hinduja S, Bartolo P. User interface tool for a novel plasma-assisted bio-additive extrusion system. Rapid Prototyping 2018 (in press).

19. Reuter S, Winter J, Schmidt-Bleker A, et al. Controlling the ambient air affected reactive species composition in the effluent of an argon plasma jet. IEEE Trans Plasma Sci 2012;40:2788-2794.

20. Liu F, Vyas C, Poologasundarampillai G, et al. Structural evolution of PCL during melt extrusion 3D printing. Macromol Mater Eng 2018;303:1700494.

21. Thakur S, Neogi S. Tailoring the adhesion of polymers using plasma for biomedical applications: A critical review. Rev Adhes Adhes 2015;3:53-97.

22. Tuzlakoglu K, Pashkuleva I, Rodrigues MT, et al. A new route to produce starch-based fiber mesh scaffolds by wet spinning and subsequent surface modification as a way to improve cell attachment and proliferation. J Biomed Mater Res A 2010;92:369-377.

23. Xu H, Hu Z, Wu S, et al. Surface modification of polytetrafluoroethylene by microwave plasma treatment of $\mathrm{H} 2$
O/Ar mixture at low pressure. Mater Chem Phys 2003; 80:278-282.

24. Wang Y, Lu L, Zheng Y, et al. Improvement in hydrophilicity of PHBV films by plasma treatment. J Biomed Mater Res Part A 2006;76:589-595.

25. Intranuovo $\mathrm{F}$, Gristina $\mathrm{R}$, Brun $\mathrm{F}$, et al. Plasma modification of PCL porous scaffolds fabricated by solvent-casting/ particulate-leaching for tissue engineering. Plasma Processes Polym 2014;11:184-195.

26. Liu F, Vyas C, Poologasundarampollai G, et al. Process driven microstructure control in melt-extrusion based $3 \mathrm{D}$ printing for tailorable mechanical properties in a filament. Macromol Mater Eng 2018 (in press).

27. Alves NM, Shi J, Oramas E, et al. Bioinspired superhydrophobic poly (L-lactic acid) surfaces control bone marrow derived cells adhesion and proliferation. J Biomed Mater Res Part A 2009;91:480-488.

Address correspondence to: Paulo Jorge Bártolo School of Mechanical, Aerospace and Civil Engineering University of Manchester Manchester M13 9PL United Kingdom

E-mail: paulojorge.dasilvabartolo@manchester.ac.uk
4 AU8

Correct 


\section{AUTHOR QUERY FOR 3DP-2018-0056-VER9-LIU_1P}

AU1: Please identify (highlight or circle) all authors' surnames for accurate indexing citations.

AU2: Please check the correctness of authors' affiliation.

AU3: Please define "PE-CVD."

AU4: Please define "WCA."

AU5: In Ref. 9, please mention the publisher location.

AU6: In Ref. 10, please mention the publisher location.

AU7: In Ref. 14, please mention the editor name and publisher location.

AU8: Please confirm the address of correspondence.

AU9: Labels (c and d) are there in Figure 2 artwork, but not mentioned in the figure legend. Please check.

AU10: Labels ( $\mathrm{d}$ and $\mathrm{e}$ ) are mentioned in the Figure 3 legend but not found in the artwork of the same. Please check. 